\title{
On the failing cases of the Johnson bound for error-correcting codes
}

\author{
Wolfgang Haas \\ Albert-Ludwigs-Universität \\ Mathematisches Institut \\ Eckerstr. 1 \\ 79104 Freiburg, Germany \\ wolfgang_haas@gmx . net
}

Submitted: Mar 4, 2008; Accepted: Apr 4, 2008; Published: Apr 18, 2008

Mathematics Subject Classification: 94B25, 94B65

\begin{abstract}
A central problem in coding theory is to determine $A_{q}(n, 2 e+1)$, the maximal cardinality of a $q$-ary code of length $n$ correcting up to $e$ errors. When $e$ is fixed and $n$ is large, the best upper bound for $A(n, 2 e+1)$ (the binary case) is the well-known Johnson bound from 1962. This however simply reduces to the sphere-packing bound if a Steiner system $S(e+1,2 e+1, n)$ exists. Despite the fact that no such system is known whenever $e \geq 5$, they possibly exist for a set of values for $n$ with positive density. Therefore in these cases no non-trivial numerical upper bounds for $A(n, 2 e+1)$ are known.

In this paper the author presents a technique for upper-bounding $A_{q}(n, 2 e+1)$, which closes this gap in coding theory. The author extends his earlier work on the system of linear inequalities satisfied by the number of elements of certain codes lying in $k$-dimensional subspaces of the Hamming Space. The method suffices to give the first proof, that the difference between the sphere-packing bound and $A_{q}(n, 2 e+1)$ approaches infinity with increasing $n$ whenever $q$ and $e \geq 2$ are fixed. A similar result holds for $K_{q}(n, R)$, the minimal cardinality of a $q$-ary code of length $n$ and covering radius $R$. Moreover the author presents a new bound for $A(n, 3)$ giving for instance $A(19,3) \leq 26168$.
\end{abstract}

\section{Introduction}

In the whole paper let $q$ denote an integer greater than one and $Q$ a set with $|Q|=q$. The Hamming distance $d(\lambda, \rho)$ between $\lambda=\left(x_{1}, \ldots, x_{n}\right) \in Q^{n}$ and $\rho=\left(y_{1}, \ldots, y_{n}\right) \in Q^{n}$ is defined by

$$
d(\lambda, \rho)=\left|\left\{i \in\{1, \ldots, n\}: x_{i} \neq y_{i}\right\}\right|
$$


Let $B_{q}(\lambda, e)$ denote the Hamming sphere with radius $e$ centered on $\lambda \in Q^{n}$,

$$
B_{q}(\lambda, e)=\left\{\rho \in Q^{n}: d(\rho, \lambda) \leq e\right\}
$$

We set

$$
V_{q}(n, e)=\left|B_{q}(\lambda, e)\right|=\sum_{0 \leq i \leq e}\left(\begin{array}{l}
n \\
i
\end{array}\right)(q-1)^{i}
$$

and

$$
\bar{V}_{q}(n, e)=\left|\left\{\rho \in Q^{n}: d(\rho, \lambda)=e\right\}\right|
$$

for any $\lambda \in Q^{n}$. Assume $d$ and $R$ are nonnegative integers. We say, that $C \subset Q^{n}$ has minimum distance at least $d$, if

$$
\forall \lambda, \rho \in C(\lambda \neq \rho \Rightarrow d(\lambda, \rho) \geq d)
$$

holds. $C \subset Q^{n}$ has covering radius at most $R$, if

$$
\forall \rho \in Q^{n} \exists \lambda \in C \quad \text { with } \quad d(\rho, \lambda) \leq \mathrm{R}
$$

holds. $A_{q}(n, d)$ denotes the maximal cardinality of a code $C \subset Q^{n}$ with minimal distance at least $d$. $K_{q}(n, R)$ denotes the minimal cardinality of a code $C \subset Q^{n}$ with covering radius at most $R$. In the binary case $q=2$ the subscript usually is omitted.

$A_{q}(n, d)$ is the most important quantity in coding theory, since $A_{q}(n, 2 e+1)$ is the maximal size of a $q$-ary code of length $n$ correcting up to $e$ errors.

Much work has been done in the last decades to give bounds for $A_{q}(n, d)$ and $K_{q}(n, R)$ (see [15], [3]). Updated internet tables are given by Brouwer [2] and Kéri [12]. Especially well-known are the sphere-packing bound

$$
A_{q}(n, 2 e+1) \leq \frac{q^{n}}{V_{q}(n, e)}
$$

and the sphere-covering bound

$$
K_{q}(n, R) \geq \frac{q^{n}}{V_{q}(n, R)}
$$

When $n$ and $e$ are comparatively small, the best upper bounds on $A_{q}(n, 2 e+1)$ usually are obtained via optimization. The Linear Programming Bound (LP) was introduced by Delsarte in (1972) [4]. Recently Schrijver [18] introduced an upper bound for $A(n, d)$, which refines the classical bound of Delsarte and is computed via semidefinite programming. Even more recently, a new SDP bound for the nonbinary case was given in [5]. However, the computation of LP and SDP bounds is not tractable for large values of $n$.

In this case the best bound is the well-known Johnson bound [9] from 1962, which improves on the sphere-packing bound. In the binary case $q=2$ a new bound was obtained by Mounits, Etzion and Litsyn [16], which always is at least as good as the Johnson bound. This bound however (like the Johnson bound) reduces to the spherepacking bound iff a Steiner system $S(e+2,2 e+2, n+1)$ exists (see [15]). A Steiner 
system $S(t, k, v)$ is a collection of $k$-subsets (blocks) of a $v$-set $S$, such that every $t$-subset of $S$ is contained in exactly one of the blocks. More information about Steiner systems can be found in every monograph on design theory, see for instance [1].

Despite the fact, that no system $S(e+2,2 e+2, n+1)$ is known whenever $e \geq 4$, they possibly exist for a set of integers $n$ of positive density when $e$ is fixed (see [15]). Therefore in these cases no nontrivial numerical upper bounds for $A(n, 2 e+1)$ are known.

In this paper the author makes use of a third method for upper-bounding $A_{q}(n, 2 e+1)$, which closes this gap in coding theory. The author extends his earlier work [6] on the system of linear inequalities satisfied by the number of elements of a code with covering radius one lying in $k$-dimensional subspaces of $Q^{n}$. In this paper the author applies a corresponding system for error-correcting codes, which in full generality is due to Quistorff [17]. The method was introduced in the late 1960s and early 1970s by Kamps, van Lint [11] and Horten, Kalbfleisch, Stanton [10], [20]. It was used in several papers, mainly for lower-bounding $K_{q}(n, R)$, see for instance Haas [6], [7], Habsieger [8], Quistorff [17] or Lang, Quistorff, Schneider [13]. Most papers deal with bounded values of $k$. Like in [6] we present an approach, where $k$ is unlimited with increasing $n$. The method is strong enough to give the first proof (to the authors best knowledge) of the following theorem.

Theorem 1. Whenever $q$ and $e \geq 2$ are fixed, then

$$
\frac{q^{n}}{V_{q}(n, e)}-A_{q}(n, 2 e+1) \rightarrow \infty \text { for } n \rightarrow \infty
$$

Since it is well-known, that $V_{q}(n, e)$ divides $q^{n}$ at most for a finite set of values for $n$ when $q$ and $e \geq 2$ are fixed (a consequence of a classical theorem of Siegel [19] on Diophantine approximation, see also [15]), Theorem 1 immediately follows from

Theorem 2. If $V_{q}(n, e)$ does not divide $q^{n}$,

$$
n \geq \exp 96
$$

and

$$
1 \leq e \leq \frac{\log n}{6(\log \log n+\log q)}
$$

then

$$
A_{q}(n, 2 e+1) \leq \frac{q^{n}}{V_{q}(n, e)}-\frac{1}{2} q^{\frac{1}{6 q} n^{\frac{1}{2 e}}}
$$

The quantities $K_{q}(n, R)$ and $A_{q}(n, d)$ are connected by the well-known Lobstein-van Wee bound (see [14] and [21])

$$
K_{q}(n, R) \geq \frac{q^{n}-A_{q}(n, 2 R+1)\left(\begin{array}{c}
2 R \\
R
\end{array}\right)}{V_{q}(n, R)-\left(\begin{array}{c}
2 R \\
R
\end{array}\right)}
$$

whenever $n \geq 2 R$, so that improved bounds on $A_{q}(n, 2 e+1)$ may lead to improved bounds on $K_{q}(n, R)$. Using (3), from Theorem 2 we derive 
Theorem 3. If $V_{q}(n, R)$ does not divide $q^{n}$,

$$
n \geq \exp 96
$$

and

$$
1 \leq R \leq \frac{\log n}{6(\log \log n+\log q)}
$$

then

$$
K_{q}(n, R) \geq \frac{q^{n}}{V_{q}(n, R)}+\frac{1}{2} q^{\frac{7}{48 q} n \frac{1}{2 R}} .
$$

From this we get

Theorem 4. Whenever $q$ and $R \geq 2$ are fixed, then

$$
K_{q}(n, R)-\frac{q^{n}}{V_{q}(n, R)} \rightarrow \infty \text { for } n \rightarrow \infty .
$$

In the binary case $q=2$ and $e=1$ we modify Theorem 3 in [7] to get a new upper bound for $A(n, 3)$, which appears to be the best known in many cases, including the case $n=4 p-1$ with a prime $p \geq 5$.

Theorem 5. If $1 \leq k \leq \frac{n+1}{2}$, then

$$
A(n, 3) \leq\left(2\left\lceil\frac{2^{n-k}+k}{n+1}\right\rceil-1-\frac{s}{k}\right) 2^{k-1}
$$

with

$$
s=\min \left\{\left\lceil\frac{2^{n-k}+k}{n+1}\right\rceil(n+1)-2^{n-k}-k ; k\right\} .
$$

Applying Theorem 5 with $n=19, k=9$ and $n=27, k=13$ gives the following

\section{Corollary 1.}

$$
\begin{aligned}
& A(19,3) \leq 26168 \quad(26208[15]), \\
& A(27,3) \leq 4792950 \quad(4793472[16]) .
\end{aligned}
$$

This paper is organized as follows. Section 2 contains some lemmas. In the sections $3,4,5$ we prove the Theorems $2,3,5$ respectively.

\section{Some Lemmas}

Lemma 1. For $1 \leq e \leq n$ we have

$$
V_{q}(n, e) \leq(q n)^{e} .
$$


Proof. Since $n-k \leq(e-k)(n-e+1)$ for $0 \leq k \leq e-1$, we get

$$
\begin{aligned}
V_{q}(n, e) & =\sum_{0 \leq i \leq e}\left(\begin{array}{c}
n \\
i
\end{array}\right)(q-1)^{i} \leq \sum_{0 \leq i \leq e}\left(\begin{array}{l}
e \\
i
\end{array}\right)(q-1)^{i}(n-e+1)^{i} \\
& =(1+(q-1)(n-e+1))^{e} \leq(q n)^{e} .
\end{aligned}
$$

Lemma 2. For $1 \leq e \leq \frac{n}{2}$ we have

$$
V_{q}(n, e-1) \leq \frac{4 e}{q n} V_{q}(n, e)
$$

Proof. Since $q \geq 2$ and $\left(\begin{array}{c}n \\ i+1\end{array}\right) /\left(\begin{array}{c}n \\ i\end{array}\right)=(n-i) /(i+1) \geq(n-e+1) / e$ for $0 \leq i \leq e-1$, we get

$$
\begin{aligned}
V_{q}(n, e) & =\sum_{0 \leq i \leq e}\left(\begin{array}{c}
n \\
i
\end{array}\right)(q-1)^{i} \\
& \geq \sum_{0 \leq i \leq e-1}\left(\begin{array}{c}
n \\
i+1
\end{array}\right)(q-1)^{i+1} \\
& \geq \frac{(q-1)(n-e+1)}{e} V_{q}(n, e-1) \\
& \geq \frac{q n}{4 e} V_{q}(n, e-1) .
\end{aligned}
$$

The next Lemma generalizes Lemma 3 in $[6]$. Here $\|\xi\|$ means the difference from $\xi$ to a nearest integer.

Lemma 3. Let $n, s, e$ be integers with $n \geq 3,1 \leq e \leq n$ and $3 e \log n+1 \leq s \leq n$. If $V_{q}(n, e)$ does not divide $q^{n}$, then there exists an integer $k$ with $s-3 e \log n \leq k \leq s$ satisfying

$$
\left\|\frac{q^{n-k}}{V_{q}(n, e)}\right\| \geq \frac{1}{2 q} .
$$

Proof. Since $V_{q}(n, e)$ does not divide $q^{n}$, we get

$$
\theta:=\left\|\frac{q^{n-s}}{V_{q}(n, e)}\right\|>0 .
$$

Let $m$ be the smallest nonnegative integer satisfying $q^{m} \theta \geq 1 /(2 q)$. We have $q^{m} \theta \leq 1 / 2$, which is obvious if $m=0$ and follows from the minimality of $m$ otherwise. This implies

$$
\left\|\frac{q^{n-k}}{V_{q}(n, e)}\right\|=\left\|q^{m} \frac{q^{n-s}}{V_{q}(n, e)}\right\|=\left\|q^{m} \theta\right\|=q^{m} \theta \geq \frac{1}{2 q}
$$


with $k:=s-m$, proving (5). Lemma 1 implies

and therefore

$$
\frac{1}{(q n)^{e}} \leq \frac{1}{V_{q}(n, e)} \leq \theta \leq \frac{1}{2 q^{m}}
$$

$$
m \leq \frac{e \log (q n)-\log 2}{\log q}<e\left(1+\frac{\log n}{\log q}\right) \leq 3 e \log n,
$$

which means $s-3 e \log n \leq k \leq s$.

Lemma 4. Let $k, r, e$ be integers with $1 \leq e \leq k$. Assume $k_{\sigma}$ is an integer for each $\sigma \in Q^{k}$. If for every $\sigma \in Q^{k}$

$$
\min _{\substack{\mu \in Q^{k} \\ d(\mu, \sigma) \leq e}} k_{\mu} \leq r-\left(k_{\sigma}-r\right) V_{q}(k, e)
$$

is satisfied, then we have

$$
\sum_{\sigma \in Q^{k}} k_{\sigma} \leq r q^{k}
$$

Proof. By (6) there is a function $f$ defined on $Q^{k}$, such that for each $\sigma \in Q^{k}$ the element $f(\sigma)=\mu \in Q^{k}$ satisfies $d(\mu, \sigma) \leq e$ and

$$
k_{\mu} \leq r-\left(k_{\sigma}-r\right) V_{q}(k, e) .
$$

We set

$$
\begin{aligned}
& A=\left\{\sigma \in Q^{k}: k_{\sigma}>r\right\}, \\
& B=\left\{\mu \in Q^{k}: \exists \sigma \in A \text { with } f(\sigma)=\mu\right\} .
\end{aligned}
$$

For $\mu \in B$ we have $k_{\mu} \leq r$ by (7) and thus $A, B$ are disjoint. For $\mu \in B$ we set

$$
A_{\mu}=\{\sigma \in A: f(\sigma)=\mu\} \cup\{\mu\} .
$$

The sets $A_{\mu}, \mu \in B$ are pairwise disjoint. For $\mu \in B$ we have $A_{\mu} \cap A \neq \emptyset$. Thus for $\mu \in B$ we may fix $\sigma_{\mu} \in A_{\mu} \cap A$ with $k_{\sigma_{\mu}}=\max _{\sigma \in A_{\mu} \cap A} k_{\sigma}$. For $\mu \in B$

$$
\begin{aligned}
\sum_{\sigma \in A_{\mu}} k_{\sigma} & =\sum_{\sigma \in A_{\mu} \cap A} k_{\sigma}+k_{\mu} \\
& \leq\left|A_{\mu} \cap A\right| k_{\sigma_{\mu}}+r-\left(k_{\sigma_{\mu}}-r\right) V_{q}(k, e) \quad \text { by }(7) \\
& \leq\left|A_{\mu} \cap A\right| k_{\sigma_{\mu}}+r-\left(k_{\sigma_{\mu}}-r\right)\left|A_{\mu} \cap A\right| \\
& =r\left(1+\left|A_{\mu} \cap A\right|\right) \\
& =r\left|A_{\mu}\right| .
\end{aligned}
$$

By $A \subset \cup_{\mu \in B} A_{\mu}$ we have $k_{\sigma} \leq r$ for $\sigma \in Q^{k}-\bigcup_{\mu \in B} A_{\mu}$. Thus

$$
\begin{aligned}
\sum_{\sigma \in Q^{k}} k_{\sigma} & =\sum_{\mu \in B} \sum_{\sigma \in A_{\mu}} k_{\sigma}+\sum_{\sigma \in Q^{k}-\bigcup_{\mu \in B} A_{\mu}} k_{\sigma} \\
& \leq r \sum_{\mu \in B}\left|A_{\mu}\right|+r\left(\sum_{\sigma \in Q^{k}} 1-\sum_{\mu \in B}\left|A_{\mu}\right|\right) \\
& =r q^{k} .
\end{aligned}
$$




\section{Proof of Theorem 2}

Without proof we first state our main tool, Quistorff's system of linear inequalities. Assume $C \subset Q^{n}$. For $\sigma \in Q^{k}, 1 \leq k \leq n$ we define

$$
\begin{aligned}
Q_{\sigma}^{n} & =\left\{\left(x_{1}, \ldots, x_{k}, \ldots, x_{n}\right) \in Q^{n}:\left(x_{1}, \ldots, x_{k}\right)=\sigma\right\} \\
k_{\sigma} & =\left|C \cap Q_{\sigma}^{n}\right| .
\end{aligned}
$$

Theorem 6 (Quistorff [17]). Assume $1 \leq e \leq k<n$. If $C \subset Q^{n}$ has minimal distance at least $2 e+1$, then for each $\sigma \in Q^{k}$ we have

$$
\sum_{0 \leq i \leq e} \sum_{\substack{\mu \in Q^{k} \\ d(\mu, \sigma)=i}} k_{\mu} V_{q}(n-k, e-i) \leq q^{n-k}
$$

For the proof of Theorem 2 let $C \subset Q^{n}$ be a code with minimal distance at least $2 e+1$ and $|C|=A_{q}(n, 2 e+1)$. We set

$$
s=\left\lfloor\frac{1}{4 q} n^{\frac{1}{2 e}}\right\rfloor .
$$

By (1) and (2) we have $\log 4 \leq \log \frac{1}{24}+\log \log n$ and $e \leq \log n$. Thus

$$
\begin{aligned}
\log 4+\log e+\log \log n & \leq \log 4+2 \log \log n \\
& \leq \log \frac{1}{24}+3 \log \log n \\
& \leq \log \frac{1}{24}-\log q+3(\log \log n+\log q) \\
& \leq \log \frac{1}{24}-\log q+\frac{1}{2 e} \log n \quad \text { by }(2) .
\end{aligned}
$$

Exponentiation yields

$$
3 e \log n+1 \leq 4 e \log n \leq \frac{1}{24 q} n^{\frac{1}{2 e}} \leq \frac{1}{4 q} n^{\frac{1}{2 e}}-1 \leq s \leq \frac{n}{2} .
$$

We therefore may apply Lemma 3 and find an integer $k$ in the interval $[s-3 e \log n, s]$, such that (5) is satisfied. By (9) we have

$$
\begin{aligned}
k-1 & \geq s-3 e \log n-1 \\
& \geq \frac{1}{4 q} n^{\frac{1}{2 e}}-2(3 e \log n+1) \\
& \geq \frac{1}{6 q} n^{\frac{1}{2 e}}
\end{aligned}
$$

and

$$
1 \leq e \leq k \leq s \leq \frac{n}{2}
$$


Moreover, since $(16 e)^{1 /(2 e)}$ is decreasing for $e \geq 1$,

$$
k \leq s \leq \frac{1}{4 q} n^{\frac{1}{2 e}} \leq \frac{1}{(16 e)^{1 /(2 e)} q} n^{\frac{1}{2 e}}
$$

which by Lemma 1 implies

$$
n \geq 16 e(q k)^{2 e} \geq 16 e V_{q}^{2}(k, e) .
$$

We now set

$$
r=\left\lfloor\frac{q^{n-k}}{V_{q}(n, e)}\right\rfloor
$$

From (5) follows

$$
r+\frac{1}{2 q} \leq \frac{q^{n-k}}{V_{q}(n, e)} \leq r+1-\frac{1}{2 q}
$$

Now consider the numbers $k_{\sigma}, \sigma \in Q^{k}$ defined in (8). We fix $\sigma \in Q^{k}$ and set

$$
N=\min _{\substack{\mu \in Q^{k} \\ d(\mu, \sigma) \leq e}} k_{\mu} \leq k_{\sigma}
$$

By (11) we may apply Theorem 6 to get

$$
\begin{aligned}
q^{n-k} & \geq \sum_{0 \leq i \leq e} \sum_{\substack{\mu \in Q^{k} \\
d(\mu, \sigma)=i}} k_{\mu} V_{q}(n-k, e-i) \\
& \geq k_{\sigma} V_{q}(n-k, e)+N \sum_{1 \leq i \leq e} \bar{V}_{q}(k, i) V_{q}(n-k, e-i) \\
& =k_{\sigma} V_{q}(n, e)-\left(k_{\sigma}-N\right) \sum_{1 \leq i \leq e} \bar{V}_{q}(k, i) V_{q}(n-k, e-i) \\
& \geq k_{\sigma} V_{q}(n, e)-\left(k_{\sigma}-N\right) V_{q}(k, e) V_{q}(n, e-1) \\
& \geq k_{\sigma} V_{q}(n, e)-\left(k_{\sigma}-N\right) \frac{4 e}{q n} V_{q}(k, e) V_{q}(n, e) \quad \text { by Lemma } 2 \\
& \geq k_{\sigma} V_{q}(n, e)-\left(k_{\sigma}-N\right) \frac{V_{q}(n, e)}{4 q V_{q}(k, e)} \quad \text { by } \quad \text { (12) }
\end{aligned}
$$

and thus

$$
r+1-\frac{1}{2 q} \geq \frac{q^{n-k}}{V_{q}(n, e)} \geq k_{\sigma}-\frac{k_{\sigma}-N}{4 q V_{q}(k, e)} .
$$

by (13). We now apply Lemma 4 . Assume $k_{\sigma}>r$. Then

$$
\frac{k_{\sigma}-r}{2 q} \leq k_{\sigma}-r-1+\frac{1}{2 q} \leq \frac{k_{\sigma}-N}{4 q V_{q}(k, e)},
$$


which is equivalent to

$$
\begin{aligned}
\min _{\substack{\mu \in Q^{k} \\
d(\mu, \sigma) \leq e}} k_{\mu}=N & \leq k_{\sigma}-2\left(k_{\sigma}-r\right) V_{q}(k, e) \\
& =r+\left(k_{\sigma}-r\right)-2\left(k_{\sigma}-r\right) V_{q}(k, e) \\
& \leq r-\left(k_{\sigma}-r\right) V_{q}(k, e) .
\end{aligned}
$$

Therefore the proposition (6) in Lemma 4 is satisfied for the numbers $k_{\sigma}$ defined in (8) (the case $k_{\sigma} \leq r$ is trivial). An application of Lemma 4 now yields

$$
\begin{array}{rlr}
A_{q}(n, 2 e+1)=|C| & =\sum_{\sigma \in Q^{k}} k_{\sigma} \\
& \leq r q^{k} \\
& \leq\left(\frac{q^{n-k}}{V_{q}(n, e)}-\frac{1}{2 q}\right) q^{k} \quad \text { by }(13) \\
& =\frac{q^{n}}{V_{q}(n, e)}-\frac{1}{2} q^{k-1} \\
& \leq \frac{q^{n}}{V_{q}(n, e)}-\frac{1}{2} q^{\frac{1}{6 q} n^{\frac{1}{2 e}}} \quad \text { by }(10),
\end{array}
$$

completing the proof of Theorem 2 .

\section{Proof of Theorem 3}

The propositions of Theorem 2 are satisfied for $e=R$ and we get

$$
A_{q}(n, 2 R+1) \leq \frac{q^{n}}{V_{q}(n, R)}-\frac{1}{2} q^{\frac{1}{6 q} n^{\frac{1}{2 R}}} .
$$

This inserted in (3) yields

$$
K_{q}(n, R) \geq \frac{q^{n}}{V_{q}(n, R)}+\frac{q^{\frac{1}{6 q} n^{\frac{1}{2 R}}}}{2 V_{q}(n, R)} .
$$

By Lemma 1 we have

$$
\begin{aligned}
V_{q}(n, R) & \leq(q n)^{R} \\
& =\exp (R \log q n) \\
& \leq \exp (2 R \log q \log n) \\
& \left.\leq \exp \left(\frac{1}{48 q} n^{\frac{1}{2 R}} \log q\right) \quad \text { by (9) (with } e=R\right) \\
& =q^{\frac{1}{48 q} n^{\frac{1}{2 R}}}
\end{aligned}
$$

and Theorem 3 follows. 


\section{$5 \quad$ Proof of Theorem 5}

Let $\mathbf{F}=\{0,1\}$ denote the finite field with two elements. We start with

Lemma 5. Let $k, l, r$ and $s$ be integers with $1 \leq k \leq l$ and $0 \leq s \leq k$. Assume the integers $x_{\sigma}, \sigma \in \boldsymbol{F}^{k}$ satisfy

$$
l x_{\sigma}+\sum_{\mu \in \boldsymbol{F}^{k}, d(\mu, \sigma)=1} x_{\mu} \leq l(r+1)+k r-s
$$

for each $\sigma \in \boldsymbol{F}^{k}$. Then

$$
\sum_{\sigma \in \boldsymbol{F}^{k}} x_{\sigma} \leq\left(2 r+1-\frac{s}{k}\right) 2^{k-1} .
$$

Proof. Put

$$
B=\left\{\sigma \in \mathbf{F}^{k}: x_{\sigma}>r\right\}, \quad N=|B| .
$$

For $\sigma \in \mathbf{F}^{k}, 1 \leq i \leq k$ and $0 \leq j \leq 2$ we define

$$
\begin{aligned}
L(\sigma, i) & =\left\{\mu \in \mathbf{F}^{k}: \mu \text { and } \sigma \text { differ at most in the } i \text { th coordinate }\right\} \\
\mathcal{L} & =\left\{L(\sigma, i): \sigma \in \mathbf{F}^{k}, 1 \leq i \leq k\right\} \\
\mathcal{L}_{j} & =\{L \in \mathcal{L}:|L \cap B|=j\} \\
y_{j} & =\left|\mathcal{L}_{j}\right|
\end{aligned}
$$

One easily gets $|L|=2$ for $L \in \mathcal{L}$ and $|\mathcal{L}|=k 2^{k-1}$. Thus we have

$$
k 2^{k-1}=|\mathcal{L}|=y_{0}+y_{1}+y_{2} .
$$

Moreover for each $\sigma \in \mathbf{F}^{k}$

$$
\sum_{L \in \mathcal{L}, \sigma \in L} 1=k
$$

Finally we define a function $g$ on $\mathcal{L}$ by

$$
g(L)=\sum_{\mu \in L} x_{\mu}-(2 r+1) \quad \text { for } L \in \mathcal{L} .
$$

We have

$$
\begin{aligned}
& \sum_{L \in \mathcal{L}} g(L)=\sum_{0 \leq j \leq 2} \sum_{L \in \mathcal{L}_{j}} g(L) \\
= & \frac{1}{2} \sum_{1 \leq j \leq 2} j \sum_{L \in \mathcal{L}_{j}} g(L)+\frac{1}{2} \sum_{L \in \mathcal{L}_{1}} g(L)+\sum_{L \in \mathcal{L}_{0}} g(L) \\
= & \frac{1}{2} \sum_{\sigma \in B} \sum_{L \in \mathcal{L}, \sigma \in L} g(L)+\frac{1}{2} \sum_{L \in \mathcal{L}_{1}} g(L)+\sum_{L \in \mathcal{L}_{0}} g(L),
\end{aligned}
$$


because in the sum $\sum_{\sigma \in B} \sum_{L \in \mathcal{L}, \sigma \in L} g(L)$ every $g(L)$ with $L \in \mathcal{L}$ and $|L \cap B|=j(j \in$ $\{1,2\})$ is counted exactly $j$ times. We now estimate the sums occurring at the right-hand side of (19). If $L \in \mathcal{L}_{0}$ we have $g(L) \leq 2 r-(2 r+1)=-1$ and thus

$$
\sum_{L \in \mathcal{L}_{0}} g(L) \leq-y_{0}
$$

If $\sigma \in B$ then

$$
\begin{aligned}
\sum_{L \in \mathcal{L}, \sigma \in L} g(L) & =\sum_{L \in \mathcal{L}, \sigma \in L} \sum_{\mu \in L} x_{\mu}-(2 r+1) k \quad \text { by }(17) \text { and }(18) \\
& =k x_{\sigma}+\sum_{\mu \in \mathbf{F}^{k}, d(\mu, \sigma)=1} x_{\mu}-(2 r+1) k \\
& =l x_{\sigma}+\sum_{\mu \in \mathbf{F}^{k}, d(\mu, \sigma)=1} x_{\mu}-(l-k) x_{\sigma}-(2 r+1) k \\
\leq & l(r+1)+k r-s-(l-k)(r+1)-(2 r+1) k \\
& \text { by }(14), l \geq k \text { and } x_{\sigma} \geq r+1 \text { for } \sigma \in B \\
= & -s
\end{aligned}
$$

implying

$$
\sum_{\sigma \in B} \sum_{L \in \mathcal{L}, \sigma \in L} g(L) \leq-N s .
$$

Furthermore, if $\sigma \in B$ and $L \in \mathcal{L} \backslash \mathcal{L}_{1}$ with $\sigma \in L$, then $L \in \mathcal{L}_{2}$ implying $g(L)>0$. Thus

$$
\sum_{L \in \mathcal{L}_{1}} g(L)=\sum_{\sigma \in B} \sum_{L \in \mathcal{\mathcal { L } _ { 1 } , \sigma \in L}} g(L) \leq \sum_{\sigma \in B} \sum_{L \in \mathcal{L}, \sigma \in L} g(L) \leq-N s
$$

by (21). Inserting this, (20) and (21) in (19) we get

$$
\sum_{L \in \mathcal{L}} g(L) \leq-N s-y_{0}
$$

Moreover by (17)

$$
k N=\sum_{\sigma \in B} \sum_{L \in \mathcal{L}, \sigma \in L} 1=y_{1}+2 y_{2} .
$$

Thus by (16) and $0 \leq s \leq k$

$$
\sum_{L \in \mathcal{L}} g(L) \leq-\frac{k N s}{k}-y_{0}=-y_{0}-\frac{s}{k} y_{1}-\frac{2 s}{k} y_{2} \leq-\frac{s}{k}\left(y_{0}+y_{1}+y_{2}\right)=-s 2^{k-1} .
$$


By (16) we now have

$$
\begin{aligned}
k \sum_{\sigma \in \mathbf{F}^{k}} x_{\sigma} & =\sum_{L \in \mathcal{L}} \sum_{\sigma \in L} x_{\sigma} \\
& =\sum_{L \in \mathcal{L}}(g(L)+2 r+1) \\
& =\sum_{L \in \mathcal{L}} g(L)+(2 r+1) k 2^{k-1} \\
& \leq-s 2^{k-1}+(2 r+1) k 2^{k-1}
\end{aligned}
$$

and (15) follows.

Proof of Theorem 5. Assume $C \subset \mathbf{F}^{n}$ is a binary code of length $n$ with minimal distance at least three and $|C|=A(n, 3)$. By Theorem 6 the numbers $k_{\sigma}, \sigma \in \mathbf{F}^{k}$ defined in (8) satisfy

$$
(n-k+1) k_{\sigma}+\sum_{\mu \in \mathbf{F}^{k}, d(\mu, \sigma)=1} k_{\mu} \leq 2^{n-k} .
$$

We now apply Lemma 5. An easy calculation shows, that (14) is satisfied for the integers $k_{\sigma}, \sigma \in \mathbf{F}^{k}$ with $l=n-k+1$,

$$
r=\left\lceil\frac{2^{n-k}+k}{n+1}\right\rceil-1
$$

and $s$ defined in (4). $k \leq l$ holds by $k \leq \frac{n+1}{2}$. Now by (15) we have

$$
A(n, 3)=|C|=\sum_{\sigma \in \mathbf{F}^{k}} k_{\sigma} \leq\left(2\left\lceil\frac{2^{n-k}+k}{n+1}\right\rceil-1-\frac{s}{k}\right) 2^{k-1} .
$$

\section{Acknowledgement}

I wish to thank Jörn Quistorff (Berlin), who informed me on his important system of linear inequalities for error-correcting codes [17], and Laurent Habsieger (Lyon), whose fine paper [8] inspired me to the present work. I am also grateful to anonymous referees for valuable remarks concerning the history of the problem and technical improvements as well as simplifications for section 2 .

\section{References}

[1] T. Beth, D. Jungnickel, H. Lenz, Design Theory, Cambridge University Press, 1999, 2nd edition. 
[2] A. Brouwer, Tables of general binary codes, http://www.win.tue.nl/ aeb/codes/binary-1.html.

[3] G. Cohen, I.S. Honkala, S. Litsyn, A. Lobstein, Covering codes, North Holland Mathematical Library, vol 54, 1997, Elsevier.

[4] Ph. Delsarte, Bounds for unrestricted codes, by linear programming, Philips Res. Repts. 27 (1972), 272-289.

[5] D. GijswiJt, A. Schrijver, H. Tanaka, New upper bounds for nonbinary codes based on the Terwilliger algebra and semidefinite programming, J. Comb. Theory, Ser. A 113 (8) (2006), 1719-1731.

[6] W. HAAs, Lower bounds for q-ary codes of covering radius one, Discr. Mathematics 219 (2000), 97-106.

[7] W. HAAS, Binary and ternary codes with covering radius one: Some new lower bounds, Discr. Mathematics 256 (2002), 161-178.

[8] L. HABsieger, Lower bounds for q-ary coverings by spheres of radius one, J. Comb. Theory Ser. A 67 (1994), 199-222.

[9] S. M. Johnson, A new upper bound for error-correcting codes, IEEE Trans. Inform. Th. 8 (1962), 203-207.

[10] J. G. Kalbfleisch, R. G. Stanton, J. D. Horten, On covering sets and errorcorrecting codes, J. Comb. Theory 11 (1971), 233-250.

[11] H. J. L. Kamps, J. H. van Lint, The football pool problem for 5 matches, J. Comb. Theory 3 (1967), 315-325.

[12] G. KÉRI, Tables for Covering Codes, http://www.sztaki.hu/ keri/codes/.

[13] W. Lang, J. Quistorff, E. Schneider, New Results on Integer Programming for Codes, Cong. Numer. 188 (2007), 97-107.

[14] A. C. Lobstein, Contributions au codage combinatoire; ordres additifs, rayon de recouvrements, Thesè, Télékom, France, (1985), 163 pp.

[15] F.J. MacWilliams, N.J.A. Sloane, The Theory of Error-Correcting Codes, Amsterdam, North-Holland, 1977.

[16] B. Mounits, T. Etzion, S. Litsyn, Improved Upper Bounds on Sizes of Codes, IEEE Trans. Inform. Th. 48 (2002), 880-886.

[17] J. QuistorfF, Improved Sphere Bounds in Finite Metric Spaces, Bull. of the ICA 46 (2006), 69-80.

[18] A. SchriJVER, New code upper bounds from the Terwilliger algebra and semidefinite programming, IEEE Trans. Inform. Th. 51 (2005), 2859-2866.

[19] C. L. Siegel, Approximation algebraischer Zahlen, Math. Zeit. 10 (1921), 173-213.

[20] R. G. Stanton, J. G. Kalbfleisch, Intersection inequalities for the covering problem, SIAM J. Appl. Math. 17 (1969), 1311-1316.

[21] G. J. M. van WeE, Some new lower bounds for binary and ternary covering codes, IEEE Trans. Inform. Th. 39 (1993), 1422-1424. 